a poorer prognosis; a Dutch team has confirmed these results. Surprisingly, they found that the mere presence of a tertiary neoplasm, independent of its Gleason grade, is associated with a shorter time to PSA progression and worse outcome, and suggest that the contribution of tumor multifocality to prognosis is under-appreciated.

The study population included men with organ-confined tumor who underwent radical retropubic prostatectomy at a single center. Clinical data and prostatectomy specimens were obtained from 223 patients, nearly half of whom had a tertiary neoplasm comprising, on average, $7 \%$ of the total tumor volume.

The 5-year risk of PSA progression was three times higher in men with a tertiary neoplasm, irrespective of its Gleason grade, compared with those without (log rank $P=0.0002)$. Multivariate analysis, however, showed that tertiary neoplasm had a smaller effect on hazard ratios $(\mathrm{HR})$ for PSA progression than either pathologic stage or positive surgical margins (HRs 1.78, 2.34 and 3.95, respectively). The presence of a tertiary neoplasm might be a useful additional factor in the assessment of a patient's overall risk of recurrence.

Caroline Barranco

Original article van Oort IM et al. (2005) Does the tertiary Gleason pattern influence the PSA progression-free interval after retropubic radical prostatectomy for organ-confined prostate cancer? Eur Urol 48: 572-576

\section{Predicting mortality after hormonal therapy for postsurgical or postradiologic PSA failure}

Around $30 \%$ of patients with localized prostate cancer who undergo radical prostatectomy or external-beam radiation therapy show PSA failure within 10 years and median survival in these patients is related to PSA doubling time. PSA doubling time prior to the initiation of primary androgen-suppression therapy (AST) has also been associated with prostate-cancerspecific mortality (PCSM), as has posthormonal PSA nadir. Although it has been shown that patients who do not achieve a post-AST nadir below $0.2 \mathrm{ng} / \mathrm{ml}$ following primary treatment have an increased risk of PCSM, it is not known whether this also applies in cases of secondary AST given for PSA failure after surgery or radiation therapy.
In this study of 747 men with rising PSA and negative bone scans after radical prostatectomy $(n=486)$ or external-beam radiation therapy ( $n=261)$, PSA nadir at 8 months postAST initiation, pre-AST PSA doubling time, PSA level and a Gleason score of 8-10 were significantly associated with time to PCSM. A PSA nadir of $>0.2 \mathrm{mg} / \mathrm{ml}$ combined with pre-AST PSA doubling time of $<3$ months was closely associated with PCSM, independent of initial prostate cancer treatment, and this profile was seen in $75 \%$ of those patients who died of prostate cancer over the observation period of the study. Given the clinical relevance of these findings, the authors suggest that these criteria are a useful endpoint for future clinical trials.

Carol Lovegrove

Original article Stewart AJ et al. (2005) Prostate-specific antigen nadir and cancer-specific mortality following hormonal therapy for prostate-specific antigen failure. J Clin Oncol 23: 6556-6560

\section{Use of $\alpha$-blocker therapy for dysfunctional voiding in children}

Biofeedback has proved useful for retraining pelvic-floor muscles and improving bladder emptying in cases of dysfunctional voiding in children with urinary retention; however, these techniques require multiple clinic visits and well-motivated parents and children. Recently, $a$-blockers have been reported to be effective in this condition and their efficacy was compared with biofeedback in this study by Yucel and colleagues.

In 28 children (mean age of 6.25 years) with symptoms of urinary incontinence, urgency and urinary tract infections, biofeedback and $a$-blocker therapies were comparable in terms of reducing postvoid residual (PVR) urine volume and improving average flow rate. This improvement persisted for 6 months after biofeedback treatment and during 6 months of continuous $a$-blocker therapy. Complete improvement in urge-incontinence episodes was seen in $62.5 \%$ and $70 \%$ of the biofeedback and $\alpha$-blocker groups, respectively. Combining $a$-blockers and biofeedback improved outcome in five out of six of those children who did not show reduced PVR urine volume after a single therapy. No adverse effects were reported in the $\alpha$-blocker group and parental satisfaction was significantly higher for $\alpha$-blockers than for biofeedback. 